\title{
SOME RESULTS CONCERNING FRAMES, ROOM SQUARES, AND SUBSQUARES
}

\author{
D. R. STINSON
}

(Received 11 August 1980)

Communicated by W. D. Wallis

\begin{abstract}
Frames have been defined as a certain type of generalization of Room square. Frames have proven useful in the construction of Room squares, in particular, skew Room squares.

We generalize the definition of frame and consider the construction of Room squares and skew Room squares using these more general frames.

We are able to construct skew Room squares of three previously unknown sides, namely 93, 159, and 237. This reduces the number of unknown sides to four: 69, 87, 95 and 123. Also, using this construction, we are able to give a short proof of the existence of all skew Room squares of (odd) sides exceeding 123.

Finally, this frame construction is useful for constructing Room squares with subsquares. We can also construct Room squares "missing" subsquares of sides 3 and 5 . The "missing" subsquares of sides 3 and 5 do not exist, so these incomplete Room squares cannot be completed to Room squares.
\end{abstract} 1980 Mathematics subject classification (Amer. Math. Soc.): 05 B 30.

\section{Introduction: Room squares}

A Room square of side $s$ is a square array $R$ of side $s$, satisfying the following properties:

(1) each cell of $R$ is either empty or contains an unordered pair of elements (symbols) chosen from a set $S$ of size $s+1$,

(2) each symbol occurs precisely once in each row and column of $R$,

(3) every unordered pair of symbols occurs in a unique cell of $R$.

The following is shown in [9]. 
THEOREM 1.1. A Room square of side $s$ exists if and only if $s$ is a positive odd integer other than 3 or 5 , or (by convention) if $s=0$.

Suppose $R$ is a Room square of side $s$, on symbol set $S$. A square $t$ by $t$ subarray of $R$ is said to be a Room subsquare of side $t$ provided it is itself a Room square (of side $t$ ). We will refer to a Room subsquare simply as a subsquare.

An incomplete Room square of side $s$ missing a subsquare of side $t$, is a square array $R$ of side $s$, which satisfies the following:

(1) each cell of $R$ is either empty or contains an unordered pair of symbols chosen from $S(|S|=s+1)$,

(2) there exists an empty square subarray $R^{\prime}$ of $R$, having side $t$,

(3) a row of column of $R$ not meeting $R^{\prime}$ contains each symbol of $S$ precisely once,

(4) a row of column of $R$ which meets $R^{\prime}$ contains precisely the symbols $S \backslash T$, where $T \subseteq S$ and $|T|=t+1$,

(5) the pairs occurring in $R$ are precisely those $\{x, y\} \subseteq(S \times S) \backslash(T \times T)$, with $x \neq y$.

For brevity, we refer to an incomplete Room square of side $s$ missing a subsquare of side $t$ as an incomplete $(s, t)$-Room square. Clearly existence requires $s$ and $t$ to be odd, or zero. Also, if $t$ is odd and not 3 or 5 , or if $t=0$, then the existence of an incomplete $(s, t)$-Room square is equivalent to the existence of a Room square of side $s$ containing a subsquare of side $t$. This is clear since the subsquare of side $t$ may be inserted or removed at will. If $t=3$ or 5 , however, an incomplete $(s, t)$-Room square cannot be completed, since the missing subsquare does not exist.

A Room square $R$, on symbol set $S$, is said to be standardized with respect to the symbol $\infty \in S$, provided the rows and columns of $R$ have been permuted (if necessary) so that $\infty$ occurs in the cells of $R$ on the main diagonal. Given a standardized Room square, it is natural to index the rows and columns of $R$ so that $\{\infty, x\}$ occurs in cell $(x, x)$ of $R$, for every $x \in S, x \neq \infty$.

A standardized Room square $R$ (of side $s$ ) is said to be a skew Room square (of side $s$ ) provided that, for any pair of cells $(i, j)$ and $(j, i)$, where $i \neq j$, precisely one is empty.

Skew Room squares have been studied extensively, and the following is known [1].

THEOREM 1.2. There exists a skew Room square of side $s$ if $s$ is odd, $s>5$, and $s \notin\{69,87,93,95,123,159,237\}$. 
We will construct skew Room squares of sides 93,159 , and 237, reducing the number of unknown cases to four.

A subsquare of a skew Room square $R$ is said to be a skew subsquare provided it is located symmetrically with respect to the main diagonal of $R$.

Suppose $R$ is an incomplete $(s, t)$-Room square. $R$ is standardized as follows. Pick an element $\infty \in T$. If necessary, permute the rows and columns of $R$ so that $R^{\prime}$ is located symmetrically with respect to the main diagonal, and so that $\infty$ occurs in the cells of $R \backslash R^{\prime}$ on the main diagonal. Choose any indexing of the rows and columns of $R$ (by $S$ ) such that $\{\infty, x\}$ occurs in cell $(x, x)$, for all $x \in S \backslash T$.

We say that a (standardized) incomplete $(s, t)$-Room square is skew provided that, of any two cells $(i, j)$ and $(j, i)$ where $j \neq i$, and $\{i, j\} \nsubseteq T \times T$, precisely one is empty. Thus if there exists a skew incomplete $(s, t)$-Room square, say $R$, and there exists a skew Room square of side $t$, then $R$ may be completed to a skew Room square of side $s$.

In the next section we define and give a construction for a general type of frame. In Section 3, we use these frames to construct some incomplete Room squares, both skew and nonskew.

\section{Frames}

Suppose $u_{i}$ and $t_{i}, 1 \leqslant i \leqslant k$, are positive integers. Let $S$ be a set of cardinality $s=\sum_{i=1}^{k} t_{i} u_{i}$, and suppose $S=\bigcup_{j=1}^{n} S_{j}$, where $n=\sum_{i=1}^{k} u_{i}$. Further, suppose the $S_{j}$ 's are pairwise disjoint, and there exist exactly $u_{i} S_{j}$ 's with cardinality $t_{i}$, for $1 \leqslant i \leqslant k$.

We define a frame of type $t_{1}^{\mu_{1}} t_{2}^{u_{2}} \cdots t_{k}^{u_{k}}$ to be a square array $F$ of side $s$ whose rows and columns are indexed by $S$, which satisfies the following:

(1) each cell of $F$ is either empty or contains an unordered pair of elements of $S$,

(2) the subsquares $S_{j} \times S_{j}$ of $F$ are empty, for $1 \leqslant j \leqslant n$,

(3) row (or column) $x$ contains precisely the symbols $S \backslash S_{j}$, where $x \in S_{j}$,

(4) the pairs occurring in $F$ are precisely those $\{x, y\} \subseteq S \times S$ such that $x \neq y$, and $\{x, y\} \nsubseteq S_{j} \times S_{j}$, for any $j, 1<j \leqslant n$.

A frame is skew if, given any pair of cells $(i, k)$ and $(k, i)$ where $i \neq k$ and $\{i, k\} \nsubseteq S_{j}$, for any $j$, precisely one is empty.

In [4], a $(t, u)$-frame is defined to be a frame of type $t^{u}$. It is clear that the existence of a Room square of side $s$ is equivalent to the existence of a $(1, s)$-frame. More generally, the existence of an incomplete $(s, t)$-Room square is equivalent to the existence of a frame of type $1^{s-t} t^{1}$. Also, the two notions of skew (for frames and Room squares) agree. 
We will describe a construction for frames which uses group-divisible designs. First, some definitions.

A pairwise balanced design (or PBD) is a pair $(X, \mathscr{B})$, where $\mathscr{B}$ is a set of subsets of $X$, called blocks, such that every unordered pair of elements of $X$ occurs in a unique block. A group-divisible design (or GDD), is a triple $(X, \mathcal{G}, \mathbb{Q})$ where $\mathcal{G}$ is a partition of $X$ into subsets called groups, and $\mathcal{Q}$ is a set of subsets of $X$ (blocks), such that $(X, \mathcal{G} \cup \mathbb{Q})$ is a PBD. A GDD is said to have group type $g_{1}^{u_{1}} \cdots g_{k}^{u_{k}}$ if there are precisely $u_{i}$ groups of size $g_{i}$, for $1<i<k$ (the $u_{i}$ 's and $g_{i}$ 's are positive integers).

THEOREM 2.1. Suppose $(X, \mathcal{G}, \mathbb{Q})$ is a GDD with group-type $g_{1}^{u_{1}} \cdots g_{k}^{u_{k}}$, and let $t$ be a positive integer. Suppose that for every block $A \in \mathbb{Q}$ there exists a $(t,|A|)$-frame. Then there exists a frame of type $\operatorname{tg}_{1}^{u_{1}} \operatorname{tg}_{2}^{u_{2}} \cdots \operatorname{tg}_{k}^{u_{k}}$.

REMARK. If all the $(t,|A|)$-frames are skew, then the resulting frame is also skew.

Proof. Let $I_{t}=\{1,2, \ldots, t\}$. We construct the desired frame $F$ on symbol set $X \times I_{t}$, with empty subsquares determined by the natural partition $X \times I_{t}=$ $\cup_{G \in G}\left(G \times I_{t}\right)$. For any block $A \in \mathbb{Q}$, there exists a $(t,|A|)$-frame $F_{A}$ on symbol set $A \times I_{t}$ having empty subsquares determined by the partition $A \times I_{t}$ $=\cup_{x \in A}\left(\{x\} \times I_{t}\right)$.

Now, choose a cell $C=((x, i),(y, j)) \in\left(X \times I_{t}\right)^{2}$ of $F$. If $\{x, y\} \subseteq G$ for some $G \in \mathcal{G}$, define $F(C)$ to be empty. If not, there is a unique $A \in \mathbb{Q}$ with $\{x, y\} \subseteq A$. Then define $F(C)=F_{A}(C)$.

It may be verified that $F$ is indeed a frame of the given type.

The following construction was given in [4]. We may derive it as a corollary to Theorem 2.1.

Corollary 2.2. Suppose $(X, \mathscr{B})$ is a $P B D$, and let $t$ be a positive integer. Suppose that, for every block $B \in \mathscr{B}$, there eixsts a $(t,|B|)$-frame. Then there exists a $(t,|X|)$-frame.

Proof. $(X,\{\{x\}, x \in X\}, \mathscr{B})$ is a GDD.

\section{Frames and Room squares}

We first describe a simple method of constructing Room squares by filling in the empty subsquares of a frame. The proof is trivial and therefore is omitted. 
THEOREM 3.1. Let $w>0$. Suppose there exists a frame $F$ of type $t_{1}^{u_{1}} \cdots t_{k}^{k_{k}}$, and suppose there exists a Room square of side $t_{i}+w$ containing a subsquare of side $w$, for $1 \leqslant i<k$. Then there exists a Room square of side $s+w$, where $s=$ $\sum_{i=1}^{k} t_{i} u_{i}$.

REMARK. If the frame $F$, and all the Room squares hypothesized above are skew, then the resulting Room square is skew.

Closely related is the following result.

THEOREM 3.2. Suppose there exists a frame of type $t_{1}^{u_{1}} \cdots t_{k}^{u_{k}}$, where $u_{k}=1$. Further, suppose there exists an incomplete $\left(t_{i}+w, w\right)$-Room square, for $1<i<$ $k-1$. Then there exists an incomplete $\left(s+w, t_{k}+w\right)$-Room square, where $s=\sum_{i=1}^{k} t_{i} u_{i}$.

In applying these constructions for Room squares, it is convenient to use truncated transversal designs. A transversal design $\operatorname{TD}(k, n)$ is a GDD with group type $n^{k}$, all of whose blocks have size $k$. It is well known that the existence of $\operatorname{aD}(n, k)$ is equivalent to the existence of $k-2$ mutually orthogonal Latin squares (MOLS) of order $n$ (for a definition, see [6]). A truncated transversal design is the GDD that results from deleting some points from a TD.

Corollary 3.3. Suppose there exists a TD $k+l, n)$ and $0<t_{i}<n$, for $1<i<l$. Let $t \geqslant 1$ and suppose there exists $a(t, k+j)$-frame if $0<j<l$. Then there exists a frame of type $n^{k} t_{1}^{1} \cdots t_{l}^{1}$.

Proof. Choose any $l$ groups, say $G_{1}, \ldots, G_{l}$, of a $\operatorname{TD}(k+l, n)$ and delete $n-t_{i}$ points from $G_{i}, \quad 1<i<l$. Then every block has size $k+j$, where $0<j<l$.

We further specialize Corollary 3.3, and apply it to the case of constructing skew Room squares.

LEMMA 3.4. There exist skew $(t, u)$-frames for $(t, u)=(4,4),(4,5),(2,8)$, and $(2,9)$.

Proof. A skew $(4,4)$-frame is given in [10]. Skew $(4,5)$ - and $(2,9)$-frames are given in [4, Theorem 3.4]. D. E. McDougall [7] has constructed the following skew $(2,8)$-frame. Consider

$$
S=\{\{1,14\},\{2,11\},\{3,15\},\{4,9\},\{5,6\},\{7,13\},\{10,12\}\},
$$


and

$$
T=\{\{7,4\},\{3,12\},\{10,6\},\{9,14\},\{1,2\},\{5,11\},\{13,15\}\},
$$

where the elements are unordered pairs of numbers of $Z_{16} . S$ and $T$ generate a $(2,8)$-frame by [4; Theorem 2.3], and it may be checked that this frame is skew.

COROLLARY 3.5. (i) Suppose there exists a TD(5,n), and let $0<t \leqslant n$. If there exists a skew Room square of side $4 n+1$ then there exists a skew incomplete $(s, 4 t+1)$-Room square, where $s=4(4 n+t)+1$. If, further, there exists a skew Room square of side $4 t+1$, then there exists a skew Room square of side s.

(ii) Suppose there exists a $T D(9, n)$, and let $0<t<n$. If there exist skew Room squares of sides $2 n+1$ and $2 t+1$, then there exists a skew Room square of side $s=2(8 n+t)+1$.

Proof. (i) In Corollary 3.3, put $k=4, l=1$. Now apply Theorem 3.2 with $w=1$ (note that any skew Room square contains a skew subsquare of side 1 ).

(ii) In Corollary 3.3 , put $k=2, l=1$, and proceed as above.

COROLlaRY 3.6. There exist skew Room squares of sides 93, 237, and 159.

Proof. First, $93=4 \cdot 23+1$, and $23=4 \cdot 5+3$. Thus put $n=5, t=3$ in Corollary 3.5(i). A TD(5, 5) exists, as do skew Room squares of sides 21 and 13.

Next, $237=4 \cdot 59+1$, and $59=4 \cdot 13+7$. The required ingredients are a TD $(5,13)$, and skew Room squares of sides 53 and 29.

Finally, $159=2 \cdot 79+1$, and $79=8 \cdot 9+7$. Put $n=9, t=7$ in Corollary 3.5(ii). A TD $(9,9)$ exists, as well as skew Room squares of sides 19 and 15 . Thus the result follows.

If we attempt to construct a skew Room square of side 69 by the above method, we fail. The following is obtained, however.

Corollary 3.7. There exists a skew incomplete $(69,5)-$ Room square.

Proof. $69=4 \cdot 17+1$ and $17=4 \cdot 4+1$, so put $n=4, t=1$ in Corollary 3.5(i). A TD $(5,4)$ exists, and likewise a skew Room square of side 17 exists. However, a skew Room square of side 5 does not exist, so we are able only to obtain the incomplete Room square of side 69.

We close this section by showing how Lemma 3.4 may be used to close the spectrum of skew Room squares. In establishing a constant $s_{0}$ such that $s>s_{0}, s$ odd, implies there exists a skew Room square of side $s$, PBD constructions are 
used. In [8], the first value of $s_{0}$ obtained is approximately 45,000 . The construction of Lemma 3.4 would enable a much better preliminary bound for $s_{0}$ to be obtained. We first need a result concerning TDs.

LEMMA 3.8. Suppose $n>8$. Then there exists $n_{1}$ such that $n-5<n_{1}<n$ and such that a $T D\left(9, n_{1}\right)$ exists.

Proof. Brouwer [3] has published a (finite) list of orders $n$ for which 7 MOLS of order $n$ are not known. The required property can be easily checked.

THEOREM 3.9. Suppose there exists a skew Room square for any odd side s, $125<s<1961$. Then there exists a skew Room square of any odd side $s>1963$.

Proof. We use Corollary 3.5(ii). Let $s>1963$ be odd, $s=2 m+1$. Write $m=8 n+t$, where $62<t<69$. By Lemma 3.8, choose $n_{1}$, such that $n-5<n_{1}$ $<n$ and such that a $T D\left(9, n_{1}\right)$ exists. Let $t_{1}=m-8 n_{1}$, so that $m=8 n_{1}+t_{1}$.

We check that $0 \leqslant t_{1}<n_{1}$. We have $t_{1}<69+8 \cdot 5=109$, and $n_{1}>n-5$ $=(m-t-40) / 8>(981-109) / 8=109>t_{1}$, since $s>1963=2 \cdot 981+1$.

By an induction assumption, skew Room squares of sides $2 n+1$ and $2 t+1$ exist, so we have the result. (Note that $2 n+1>2 t+1>125$.)

Thus a preliminary bound of under 2000 may be obtained for $s_{0}$, assuming that the initial segment from 125 to $s_{0}$ can be dealt with, as it can be.

\section{Incomplete Room square}

We now apply our construction to find classes of incomplete Room squares.

LEMMA 4.1. There exist $(2, u)$-frames for $u=5,6$, and 7 .

ProOF. See [12].

COROLlary 4.2. Suppose there exists a TD(7,n), and let $0<t_{i}, t<n, t_{1}>3$. Then there exists an incomplete $(s, t)$-Room square, where $s=2\left(5 n+t_{1}+t\right)+$ 1 .

Proof. Corollary 3.3 yields a frame of type $2 n^{5} 2 t^{1} 2 t_{1}^{1}$, by setting $k=5, l=2$. Apply Theorem 3.1 with $w=1$, noting that a Room square of order $m$ exists if $m$ is odd and not 3 or 5 (Theorem 1.1). The result is obtained. 
We use the following result on TDs, found in Brouwer [3].

LeMMA 4.3. There exists a $\operatorname{TD}(7, n)$ if $n>63$.

THEOREM 4.4. If $s>\max \{u+644,6 u+9\}$, with $s, u$ odd, then there exists an incomplete $(s, u)$-Room square.

Proof. Let $s=2 m+1, u=2 t+1$. Write $m=5 n+t_{1}+t$, with $3<t_{1}<7$. We must check that $n>\max \left\{63, t, t_{1}\right\}$. We have $s>u+644$, so $2 m+1>2 t$ +645 , and $m>t+322$. Now $n>\left(m-t_{1}-t\right) / 5>(m-7-t) / 5>315 / 5$ $=63$.

Also, we have $s>6 u+9$. Thus $2 m+1>12 t+15$, or $m>6 t+7$. Now $n=\left(m-t_{1}-t\right) / 5 \geqslant(m-7-t) / 5>(6 t+7-7-t) / 5=t$.

Finally, $n \geqslant 63$ and $t_{1} \leqslant 7$, so $n \geqslant t_{1}$. Corollary 4.2 yields the desired incomplete $(s, u)$-Room square.

Of course, Theorem 4.4 could be improved, given any particular value of $u$, by examining individually the orders $s$ less than $\max \{u+644,6 u+9\}$. For small values of $u$, the term $u+644$ term dominates. The existence of many $\operatorname{TD}(7, n)$ with $n<63$ indicates that the bound could very well be improved.

We state another result similar to Theorem 4.4.

THEOREM 4.5. If $s>\max \{u+868,5 u+16\}$, with $s, u \equiv 1 \bmod 4$, then there exists an incomplete $(s, u)$-Room square.

Proof. Start with $(4, u)$-frames for $u=4,5$, and 6 , which are shown to exist in [5]. Proceed as in Corollary 4.2 and Theorem 4.4, using the fact that a $\operatorname{TD}(6, n)$ exists if $n \geqslant 53$ ([3]).

We have lowered the factor of $u$ from 6 to 5 , at the expense of increasing the constant $c$ in the term $u+c$.

We finish by giving a construction for skew incomplete Room squares based on the construction of Corollary 3.7.

THEOREM 4.6. If $n \neq 1,2,3,6$ or 17 , then there exists a skew incomplete $(16 n+5,5)$-Room square.

Proof. It is known [2] that, if $n \neq 1,2,3$, or 6 , then there exist two MOLS of order $n$ containing a common transversal. The corresponding $\operatorname{TD}(4, n)$ has a set of $n$ blocks which contain each point exactly once. Adjoin a new point $\infty$ to these blocks, and create a new group $\{\infty\}$. This yields a GDD with group type 
$n^{4} 1^{1}$ and blocks of size 4 and 5. Theorem 2.1, with $t=4$, yields a skew frame of type $4 n^{4} 4^{1}$. Now apply Theorem 3.2 , with $w=1$. This yields the desired skew incomplete Room square provided a skew Room square of side $4 n+1$ exists. Thus, by Theorem 1.2 and Corollary 3.6, we are done, since $n \neq 17$.

\section{Remarks}

A generalization of frames has yielded a new construction for Room squares and skew Room squares using GDDs. Several applications are considered in this paper.

The utility of this GDD construction lies in the fact that we may use truncated transversal designs, which contain block sizes which are two consecutive integers. Previous GDD constructions (see [8]) have used GDDs where the block sizes can only be odd integers exceeding 5 (that is sides of (skew) Room squares). The greater flexibility allowed by the use of truncated TDs yields good bounds much more easily than with the older GDD constructions.

Finally, we wish to note that a skew $(2,6)$-frame, if it exists, could be used to construct skew Room squares of sides 87,95 , and 123, leaving only side 69 in doubt. The existence of a skew $(2,6)$-frame is not known, however.

\section{References}

1. B. A. Anderson, R. C. Mullin and D. R. Stinson, 'A few more skew Room squares', Utilitas Math., submitted.

2. R. K. Brayton, D. Coppersmith and A. J. Hoffman, 'Self-orthogonal Latin squares of all orders $n \neq 2,3,6$, Bull. Amer. Math. Soc. 80 (1974), 116-118.

3. A. E. Brouwer, 'The number of mutually orthogonal Latin squares-a table up to order 10000', Research Report ZW 123/79, Mathematisch Centrum, Amsterdam, 1979.

4. J. H. Dinitz and D. R. Stinson, 'The construction and uses of frames', Ars Combinatoria 10, to appear.

5. J. H. Dinitz and D. R. Stinson, 'Further results on frames', Ars Combinatoria, submitted.

6. M. Hall, Jr., Combinatorial theory (Blaisdell, Waltham, Mass. 1967).

7. D. E. McDougall (private communication).

8. R. C. Mullin, D. R. Stinson and W. D. Wallis, "Concerning the spectrum of skew Room squares', Ars Combinatoria 6 (1978), 277-291.

9. R. C. Mullin and W. D. Wallis, 'The existence of Room squares', Aequationes Math. 13 (1975), 1-7.

10. D. R. Stinson, 'A skew Room square of order 129', Discrete Math. 31 (1980), 333-335.

Department of Combinatorics \& Optimization

University of Waterloo

Waterloo, Ontario

Canada 